\title{
A Note on Discontinuous Functions with Continuous Second Iterate
}

\author{
Pingping Zhang1, Xiaobing Gong ${ }^{2}$ \\ ${ }^{1}$ Department of Mathematics, Binzhou University, Shandong, China \\ ${ }^{2}$ Department of Mathematics, Neijiang Normal University, Sichuan, China \\ Email: zhangpingpingmath@163.com, xbgong@163.com
}

Received 6 May 2015; accepted 26 June 2015; published 30 June 2015

Copyright (C) 2015 by authors and Scientific Research Publishing Inc.

This work is licensed under the Creative Commons Attribution International License (CC BY).

http://creativecommons.org/licenses/by/4.0/

(c) (i) Open Access

\section{Abstract}

This paper investigates four classes of functions with a single discontinuous point. We give the sufficient and necessary conditions under which the second order iterates are continuous functions. Furthermore, the sufficient conditions for the continuity of the even order iterates with finitely many discontinuous points are obtained.

\section{Keywords}

\section{Iteration, Discontinuous Point, Continuous Function}

\section{Introduction}

For a nonempty set $X$ and $n \in \mathbb{N}$, the $n$-th iterate of a self-mapping $f: X \rightarrow X$ is defined by $f^{n}(x)=f\left(f^{n-1}(x)\right)$ and $f^{0}(x)=x$ for all $x \in X$ inductively. As a nonlinear operator, iteration usually amplifies the complexity of functions [1]-[7], computing the $n$-th iterate of functions is complicated, even for simple functions (see [8][12]). On the other hand, iteration can turn complex functions into simple ones. Recently, the following problem was first formulated by X. Liu, L. Liu and W. Zhang: what are discontinuous functions whose iterates of a certain order are continuous? This question, together with three classes of discontinuous functions defined on compact interval, was answered in the affirmative in [13]. That is, suppose that $f:[0,1] \rightarrow[0,1]$ with a single discontinuous point (removable discontinuous point, jumping discontinuous or oscillating discontinuous), the authors respectively gave the sufficient and necessary conditions under which the second order iterates are continuous functions.

The purpose of this paper is to study the discontinuous functions defined on open interval. For four classes of discontinuous functions with unique discontinuous point, we obtain the sufficient and necessary conditions for 
functions being continuous ones under second iterate, which are easily verified respectively. As corollaries, the sufficient conditions for the continuity of the even order iterates with finitely many discontinuous points are obtained. Our results are illustrated by examples in Section 3 .

\section{Main Results}

In this section the main results for the continuity of $f^{2}$ are stated. Throughout the paper we let $I:=(0,1)$.

Theorem 1. Suppose that $f: I \rightarrow I$ has unique removable discontinuous point $x_{r}$. Let

$$
c_{r}:=f\left(x_{r}\right) \text { and } y_{r}:=\lim _{x \rightarrow x_{r}} f(x) .
$$

Then $f^{2}$ is continuous on $I$ if and only if the following conditions are fulfilled:

(A) $f\left(c_{r}\right)=\lim _{x \rightarrow y_{r}} f(x)$,

(A) $x_{r} \notin f\left(I \backslash x_{r}\right)$.

Proof. $(\Rightarrow)$ Assume that $f^{2}$ is continuous on $I$, the removable discontinuous point $x_{r}$ of $f$ is continuous point of $f^{2}$ under iteration. Whether $y_{r}$ defined by (1) is continuous point of $f$ or not, we have

$$
\lim _{x \rightarrow x_{r}} f^{2}(x)=\lim _{x \rightarrow y_{r}} f(x) .
$$

On the other hand, using the definition of $c_{r}$ and the continuity of $f^{2}$,

$$
f\left(c_{r}\right)=f^{2}\left(x_{r}\right)=\lim _{x \rightarrow x_{r}} f^{2}(x) .
$$

Thus (2) and (3) lead to $\left(\mathrm{A}_{1}\right)$. For an indirect proof of $\left(\mathrm{A}_{2}\right)$, assume that $x_{r}=f(\tilde{x})$ for $\tilde{x} \in I \backslash x_{r}$. Then

$$
\lim _{x \rightarrow \tilde{x}} f^{2}(x)=\lim _{x \rightarrow x_{r}} f(x) \neq f\left(x_{r}\right)=f^{2}(\tilde{x}),
$$

which contradicts the continuity of $f^{2}$ on $I$ and gives a proof to $\left(\mathrm{A}_{2}\right)$.

$(\Leftarrow)$ It follows from $\left(\mathrm{A}_{1}\right)$

$$
\lim _{x \rightarrow x_{r}} f^{2}(x)=\lim _{x \rightarrow y_{r}} f(x)=f\left(c_{r}\right)=f^{2}\left(x_{r}\right),
$$

implying that $f^{2}$ is continuous at $x_{r}$. The condition $\left(\mathrm{A}_{2}\right)$, i.e., $\quad x_{r} \notin f\left(I \backslash x_{r}\right)$, shows that all points $x \in I \backslash x_{r}$ are continuous points of $f^{2}$. Therefore $f^{2}$ is continuous on $I$. This completes the proof.

Corollary 1. Suppose that $f: I \rightarrow I$ has finitely many removable discontinuous points $x_{1}, x_{2}, \cdots, x_{m}$. If the following conditions

( $\left.\overline{\mathrm{A}}_{1}\right) \quad f\left(c_{i}\right)=\lim _{x \rightarrow y_{i}} f(x)$,

$\left(\overline{\mathrm{A}}_{2}\right) x_{i} \notin f\left(I \backslash\left\{x_{1}, x_{2}, \cdots, x_{m}\right\}\right)$,

are fulfilled for all $i=1,2, \cdots, m$, where $c_{i}:=f\left(x_{i}\right), y_{i}:=\lim _{x \rightarrow x_{i}} f(x)$. Then $f^{2 n}$ is continuous on I for arbitrary integer $n \geq 1$.

Proof. By using the sufficiency of Theorem 1 , the assumption $\left(\overline{\mathrm{A}}_{1}\right)$ implies that $f^{2}$ is continuous on those points $x_{1}, x_{2}, \cdots, x_{m}$ and $\left(\overline{\mathrm{A}}_{2}\right)$ guarantees that all points $x \in I \backslash\left\{x_{1}, x_{2}, \cdots, x_{m}\right\}$ are continuous points of $f^{2}$. Thus $f^{2}$ is continuous on $I$. Since the composition of continuous functions is continuous, we get the continuity of $f^{2 n}$ for all integers $n \geq 1$ inductively. This completes the proof.

Theorem 2. Suppose that $f: I \rightarrow I$ has unique jumping discontinuous point $x_{j}$. Let $c_{j}:=f\left(x_{j}\right)$ and

$$
y_{j-0}:=\lim _{x \rightarrow x_{j-0}} f(x) \text { and } y_{j+0}:=\lim _{x \rightarrow x_{j+0}} f(x) .
$$

Then $f^{2}$ is continuous on $I$ if and only if the following conditions are fulfilled:

(B) $f\left(c_{j}\right)=\lim _{x \rightarrow y_{j-0}} f(x)=\lim _{x \rightarrow y_{j+0}} f(x)$,

(B) $x_{j} \notin f\left(I \backslash x_{j}\right)$. 
Proof. $\left(\Rightarrow\right.$ ) In view of the definitions of $c_{j}, y_{j-0}, y_{j+0}$ and the continuity of $f^{2}$, we get

$$
f\left(c_{j}\right)=f^{2}\left(x_{j}\right)=\lim _{x \rightarrow x_{j-0}} f^{2}(x)=\lim _{x \rightarrow y_{j-0}} f(x),
$$

and

$$
f\left(c_{j}\right)=f^{2}\left(x_{j}\right)=\lim _{x \rightarrow x_{j+0}} f^{2}(x)=\lim _{x \rightarrow y_{j+0}} f(x) .
$$

Clearly, (4) and (5) yield $\left(\mathrm{B}_{1}\right)$. Suppose the contrary to (ii), there is $x_{j}=f(\bar{x})$ for some $\bar{x} \in I \backslash x_{j}$. The limit

$$
\lim _{x \rightarrow \bar{x}} f^{2}(x)=\lim _{x \rightarrow x_{j}} f(x)
$$

is nonexistence since $x_{j}$ is a jumping discontinuous point of $f$, which contradicts the fact that $f^{2}$ is continuous at the point $\bar{x}$. This contradiction proves $\left(\mathrm{B}_{2}\right)$.

$(\Leftarrow)$ The condition $\left(\mathrm{B}_{1}\right)$ implies

$$
\lim _{x \rightarrow x_{j-0}} f^{2}(x)=\lim _{x \rightarrow y_{j-0}} f(x)=f\left(c_{j}\right)=f^{2}\left(x_{j}\right),
$$

and

$$
\lim _{x \rightarrow x_{j+0}} f^{2}(x)=\lim _{x \rightarrow y_{j+0}} f(x)=f\left(c_{j}\right)=f^{2}\left(x_{j}\right) .
$$

Thus, (6) and (7) lead to

$$
\lim _{x \rightarrow x_{j}} f^{2}(x)=f^{2}\left(x_{j}\right),
$$

which implies that the jumping discontinuous point $x_{j}$ of $f$ change into the continuous point of $f^{2}$. Using the similar argument as the sufficiency for $\left(\mathrm{B}_{2}\right)$ in Theorem 1, we can prove that all points $x \in I \backslash x_{j}$ are continuous points of $f^{2}$. Thus $f^{2}$ is continuous on $I$. That is, we prove the sufficiency. This completes the proof.

Corollary 2. Suppose that $f: I \rightarrow I$ has finitely many jumping discontinuous points $x_{1}, x_{2}, \cdots, x_{m}$. If the following conditions

$$
\begin{aligned}
& \left(\overline{\mathbf{B}}_{1}\right) \quad f\left(c_{i}\right)=\lim _{x \rightarrow y_{i-0}} f(x)=\lim _{x \rightarrow y_{i+0}} f(x), \\
& \left(\overline{\mathbf{B}}_{2}\right) x_{i} \notin f\left(I \backslash\left\{x_{1}, x_{2}, \cdots, x_{m}\right\}\right),
\end{aligned}
$$

are fulfilled for all $i=1,2, \cdots, m$, where $c_{i}:=f\left(x_{i}\right), \quad y_{i-0}:=\lim _{x \rightarrow x_{i}-0} f(x), \quad y_{i+0}:=\lim _{x \rightarrow x_{i}+0} f(x)$. Then $f^{2 n}$ is continuous on I for arbitrary integer $n \geq 1$.

Proof. The discussion is similar as that of Corollary 1 . By using the sufficiency of Theorem 2, the assumption $\left(\overline{\mathrm{B}}_{1}\right)$ implies that $f^{2}$ is continuous on those points $x_{1}, x_{2}, \ldots, x_{m}$ and $\left(\overline{\mathrm{B}}_{2}\right)$ implies that $x \in I \backslash\left\{x_{1}, x_{2}, \cdots, x_{m}\right\}$ are all continuous points of $f^{2}$. Thus $f^{2}$ is continuous on $I$. Consequently, we obtain the continuity of $f^{2 n}$ for all integers $n \geq 1$ inductively. This completes the proof.

Theorem 3. Suppose that $f: I \rightarrow I$ has unique oscillating discontinuous point $x_{o}$. Then $f^{2}$ is continuous on I if and only if the following conditions are fulfilled:

(C) $f^{2}(x) \equiv f^{2}\left(x_{o}\right)$ on a neighborhood $U\left(x_{o}\right)$,

(C) $x_{0} \notin f\left(I \backslash x_{0}\right)$.

Proof. $(\Rightarrow)$ We first show that the condition $\left(\mathrm{C}_{1}\right)$ holds. Suppose the contrary, for any $\delta>0$ there exists a corresponding point $y_{\delta} \in U\left(x_{o}, \delta\right)$ satisfying $f^{2}\left(y_{\delta}\right) \neq f^{2}\left(x_{o}\right)$. Put $\varepsilon_{0}=\left|f^{2}\left(y_{\delta}\right)-f^{2}\left(x_{o}\right)\right|$, then for $\delta>0$ there is $y_{\delta} \in U\left(x_{o}, \delta\right)$ such that $\left|f^{2}\left(y_{\delta}\right)-f^{2}\left(x_{o}\right)\right|=\varepsilon_{0}$, implying $f^{2}$ is discontinuous at $x_{o}$, a contradiction. This gives a proof to $\left(\mathrm{C}_{1}\right)$. To prove $\left(\mathrm{C}_{2}\right)$, by reduction to absurdity, we assume that $x_{o} \in f\left(I \backslash x_{o}\right)$, there is $\hat{x} \in I \backslash x_{o}$ such that $x_{o}=f(\hat{x})$. Note that $x_{o}$ is oscillating discontinuous point of $f$, the limit

$$
\lim _{x \rightarrow \tilde{x}} f^{2}(x)=\lim _{x \rightarrow x_{o}} f(x)
$$


is nothingness, which contradicts the continuity of $f^{2}$. Therefore, the claim $\left(\mathrm{C}_{2}\right)$ is proved.

$(\Leftarrow)$ From the assumption $\left(\mathrm{C}_{1}\right)$ we see that

$$
\lim _{x \rightarrow x_{o}} f^{2}(x)=\lim _{x \rightarrow x_{o}} f^{2}\left(x_{o}\right)=f^{2}\left(x_{o}\right),
$$

implying the oscillating discontinuous point $x_{o}$ of $f$ is a continuous point of $f^{2}$. On the other hand, one can use the similar argument as the sufficiency for the condition $\left(\mathrm{C}_{2}\right)$ in Theorem 1 and prove that all points $x \in I \backslash x_{o}$ are continuous points of $f^{2}$. This completes the proof. $\square$

Corollary 3. Suppose that $f: I \rightarrow I$ has finitely many oscillating discontinuous points $x_{1}, x_{2}, \cdots, x_{m}$. If the following conditions

$\left(\overline{\mathbf{C}}_{1}\right) f^{2}(x) \equiv f^{2}\left(x_{i}\right)$ on a neighborhood $U\left(x_{i}\right)$,

$\left(\overline{\mathbf{C}}_{2}\right) x_{i} \notin f\left(I \backslash\left\{x_{1}, x_{2}, \cdots, x_{m}\right\}\right)$,

are fulfilled for all $i=1,2, \cdots, m$. Then $f^{2 n}$ is continuous on I for arbitrary integer $n \geq 1$.

Proof. The discussion is similar as that of Corollary 1 . By using the sufficiency of Theorem 3 , the second iterate $f^{2}$ is continuous on $x_{1}, x_{2}, \cdots, x_{m}$ by $\left(\overline{\mathrm{C}}_{1}\right)$ and is continuous on all points $x \in I \backslash\left\{x_{1}, x_{2}, \cdots, x_{m}\right\}$ from $\left(\overline{\mathrm{C}}_{2}\right)$, thus $f^{2}$ is continuous on $I$. Consequently, we have the continuity of $f^{2 n}$ for all integers $n \geq 1$ inductively. This completes the proof.

Theorem 4. Suppose that $f: \mathbb{R} \rightarrow \mathbb{R}$ has unique infinite discontinuous point $x_{i}$. Then $f^{2}$ is continuous on $\mathbb{R}$ if and only if the following conditions are fulfilled:

(D) $f^{2}\left(x_{i}\right)=\lim _{x \rightarrow \infty} f(x)=$ constant,

(D) $x_{i} \notin f\left(\mathbb{R} \backslash x_{i}\right)$.

Proof. $(\Rightarrow)$ Note that $f^{2}$ is continuous on $\mathbb{R}$, then the infinite discontinuous point $x_{i}$ of $f$ is a continuous point of $f^{2}$, i.e.,

$$
f^{2}\left(x_{i}\right)=\lim _{x \rightarrow x_{i}} f^{2}(x)=\lim _{x \rightarrow \infty} f(x),
$$

which shows the $\operatorname{limit}_{\lim } f(x)$ exists and is equivalent to $f^{2}\left(x_{i}\right)$. This implies the result $\left(\mathrm{D}_{1}\right)$. To prove $\left(\mathrm{D}_{2}\right)$, suppose the contrary, there exists a point $\breve{x} \in \mathbb{R} \backslash x_{i}$ such that $x_{i}=f(\breve{x})$. Since $x_{i}$ is infinite discontinuous point of $f$, the limit

$$
\lim _{x \rightarrow \breve{x}} f^{2}(x)=\lim _{x \rightarrow x_{i}} f(x)
$$

is infinite, which contradicts the continuity of $f^{2}$. Thus, the necessary proof of $\left(\mathrm{D}_{2}\right)$ is completed.

$(\Leftarrow)$ From the assumption $\left(D_{1}\right)$ and the fact $\lim _{x \rightarrow x_{i}} f^{2}(x)=\lim _{x \rightarrow \infty} f(x)$, one can see that

$$
f^{2}\left(x_{i}\right)=\lim _{x \rightarrow x_{i}} f^{2}(x)=A,
$$

implying the infinite discontinuous point $x_{i}$ of $f$ is a continuous point of $f^{2}$. If $\left(\mathrm{D}_{2}\right)$ holds, then all real numbers $x \neq x_{i}$ are continuous points of $f^{2}$. This completes the proof.

Corollary 4. Suppose that $f:(a,+\infty) \rightarrow(a,+\infty)($ or $f:(-\infty, b) \rightarrow(-\infty, b))$ has unique infinite discontinuous point $x_{i}$, where $a, b \in \mathbb{R}$. Then $f^{2}$ is continuous on $(a,+\infty)($ or $(-\infty, b))$ if and only if the following conditions are fulfilled:

$$
\begin{aligned}
& \text { ( } \left.\tilde{\mathbf{D}}_{1}\right) f^{2}\left(x_{i}\right)=\lim _{x \rightarrow+\infty} f(x)=\text { constant }\left(\text { or } f^{2}\left(x_{i}\right)=\lim _{x \rightarrow-\infty} f(x)=\text { constant }\right), \\
& \text { ( } \left.\tilde{\mathbf{D}}_{2}\right) x_{i} \notin f\left((a,+\infty) \backslash x_{i}\right)\left(\text { or } x_{i} \notin f\left((-\infty, b) \backslash x_{i}\right)\right) \text {. }
\end{aligned}
$$

Proceeding similarly as Theorem 4 one can show this corollary.

Corollary 5. Suppose that $f: \mathbb{R} \rightarrow \mathbb{R}$ has finitely many infinite discontinuous points $x_{1}, x_{2}, \cdots, x_{m}$. If the following conditions

$$
\begin{aligned}
& \left(\overline{\mathbf{D}}_{1}\right) f^{2}\left(x_{i}\right)=\lim _{x \rightarrow \infty} f(x)=\text { constant, } \\
& \left(\overline{\mathbf{D}}_{2}\right) x_{i} \notin f(\mathbb{R}),
\end{aligned}
$$


are fulfilled for all $i=1,2, \cdots, m$. Then $f^{2 n}$ is continuous on $\mathbb{R}$ for arbitrary integer $n \geq 1$.

Proof. We obtain the result by using the similar argument as Corollary 1 . In view of the sufficiency of Theorem 4, the second iterate $f^{2}$ is continuous on those points $x_{1}, x_{2}, \cdots, x_{m}$ from $\left(\overline{\mathrm{D}}_{1}\right)$ and is continuous on all points $x \in I \backslash\left\{x_{1}, x_{2}, \cdots, x_{m}\right\}$ from $\left(\overline{\mathrm{D}}_{2}\right)$, thus $f^{2}$ is continuous on $I$. Then we have the continuity of $f^{2 n}$ for all integers $n \geq 1$ inductively. This completes the proof.

\section{Examples}

In this section we demonstrate our theorems with examples.

Example 1. Consider the mapping $F_{1}:(-3,3) \rightarrow(-3,3)$ defined by

$$
F_{1}(x)= \begin{cases}\frac{1}{2} x+2, & x \in(-3,-2), \\ -1, & x=-2, \\ x+3, & x \in(-2,-1], \\ 2, & x \in(-1,3) .\end{cases}
$$

Clearly, $x_{1}=-2$ is the unique removable discontinuous point of $F_{1}$. By simple calculation, we have

$$
\begin{gathered}
c_{1}:=F_{1}(-2)=-1, \quad y_{1}:=\lim _{x \rightarrow-2} F_{1}(x)=1, \\
F_{1}\left(c_{1}\right)=\lim _{x \rightarrow y_{1}} F_{1}(x)=2 .
\end{gathered}
$$

Moreover, the set $F_{1}((-3,3) \backslash\{-2\}) \subset\left(\frac{1}{2}, 2\right] \cup\{-1\}$ is not include the point -2 . By using the sufficiency of Theorem 1, we obtain the continuity of $F_{1}^{2}$ on $(-3,3)$.

Example 2. Consider the mapping $F_{2}:(-3,4) \rightarrow(-3,4)$ defined by

$$
F_{2}(x)= \begin{cases}\frac{1}{2} x+\frac{7}{2}, & x \in(-3,-1], \\ \frac{3}{5} x+\frac{21}{10}, & x \in\left(-1, \frac{3}{2}\right], \\ -6 x+12, & x \in\left(\frac{3}{2}, 2\right], \\ 3 x-6, & x \in(2,4) .\end{cases}
$$

Clearly, $x_{2}=-1$ is the unique jumping discontinuous point of $F_{2}$. By calculating we have

$$
\begin{gathered}
c_{1}:=F_{2}(-1)=3, \quad y_{1-0}:=\lim _{x \rightarrow-1-0} F_{2}(x)=3, \quad y_{1+0}:=\lim _{x \rightarrow-1+0} F_{2}(x)=\frac{3}{2}, \\
F_{2}\left(c_{1}\right)=\lim _{x \rightarrow y_{1-0}} F_{2}(x)=\lim _{x \rightarrow y_{1+0}} F_{2}(x)=3,
\end{gathered}
$$

and $F_{2}((-3,4) \backslash\{-1\})=[0,6)$ is not include the points -1 . Then $F_{2}^{2}$ is continuous on $(-3,4)$ using the sufficiency of Theorem 2 .

Example 3. Consider the mapping $F_{3}:(4,8) \rightarrow(4,8)$ defined by

$$
F_{3}(x)= \begin{cases}7-\sin 2, & x \in\left(4, \frac{9}{2}\right], \\ 7+\sin \frac{1}{x-5}, & x \in\left(\frac{9}{2}, 6\right), \\ 7+\sin 1, & x \in[6,8) .\end{cases}
$$


Clearly, $x_{3}=5$ is an oscillating discontinuous point of $F_{3}$. By calculating, $F_{3}((4,8) \backslash\{5\}) \subset[6,8]$ is not include 5. Moreover, $F_{3}^{2}(x)=F_{3}^{2}(5)=7+\sin 1$ for $x \in\left(\frac{9}{2}, 6\right)$. Thus the function $F_{3}$ is continuous on $(4,8)$ by the sufficiency of Theorem 3.

Example 4. Consider the mapping $F_{4}:(-\infty,+\infty) \rightarrow(-\infty,+\infty)$ defined by

$$
F_{4}(x)=-\frac{1}{|x-2|} \text {. }
$$

Clearly, $x_{4}=2$ is an infinite discontinuous point of $F_{4}$. By calculating we have

$$
F_{4}^{2}(2)=\lim _{x \rightarrow \infty} F_{4}(x)=0
$$

and $F_{4}((-\infty,+\infty) \backslash\{2\}) \subseteq(-\infty, 0)$ is not include 2 , then $F_{4}$ is continuous on $(-\infty,+\infty)$ by the sufficiency of Theorem 4.

\section{Acknowledgements}

We thank the Editor and the referee for their comments. Project supported by Shandong Provincial Natural Science Foundation of China (ZR2014AL003), Scientific Research Fund of Sichuan Provincial Education Departments (12ZA086), Scientific Research Fund of Shandong Provincial Education Department (J12L59) and Doctoral Fund of Binzhou University (2013Y04).

\section{References}

[1] Lesmoir-Gordon, N. and Edney, W. (2006) Introducing Fractal Geometry. Icon Books, Cambridge.

[2] Lesmoir-Gordon, N. (2010) The Colours of Infinity: The Beauty and Power of Fractals. Springer, London. http://dx.doi.org/10.1007/978-1-84996-486-9

[3] Mandelbrot, B. (2004) Fractals and Chaos: The Mandelbrot Set and Beyond. Springer, New York. http://dx.doi.org/10.1007/978-1-4757-4017-2

[4] Zhang, P.P. (2012) Formal and Analytic Solutions for a Quadric Iterative Functional Equation. Electronic Journal of Differential Equations, 46, 1-9.

[5] Zhang, P. and Gong, X. (2014) Existence of Solutions for Iterative Differential Equaitons. Electronic Journal of Differential Equations, 7, 1-10.

[6] Zhang, P. and Mi, L. (2009) Analytic Solutions of a Second Order Iterative Functional Differential Equation. Applied Mathematics and Computation, 210, 277-283. http://dx.doi.org/10.1016/j.amc.2008.12.007

[7] Zhang, P. and Zhang, Q. (2010) Local Invertible Analytic Solutions of a First Order Iterative Functional Differential Equation. Acta Mathematica Sinica Chinese Series, 53, 409-416.

[8] Sun, D. (2004) Iteration of Quasi-Polynomial of Degree Two (in Chinese). J. Math., 24, 237-240.

[9] Targonski, G. (1981) Topics in Iterative Theory. Vandenhoeck and Ruprecht, Götingen.

[10] Wu, Z. and Sun, D. (2006) The Iteration of Quasi-Polynomials mappings (in Chinese). Acta Math. Sci. A., 26, 493-497.

[11] Xu, L. and Xu, S. (2006) On Iteratio of Linear Fractional Function and Applications (in Chinese). Math. Pract. Theory., 36, 225-228.

[12] Yu, Z., Yang, L. and Zhang, W. (2012) Discussion on Polynomials Having Polynomial Iterative Roots. Journal of Symbolic Computation, 47, 1154-1162. http://dx.doi.org/10.1016/j.jsc.2011.12.038

[13] Liu, X., Liu, L. and Zhang, W. Discontinuous Function with Continuous Second Iterate. Aequat. Math. 